\title{
Delay Differential Equations in Biosciences: Parameter estimation and sensitivity analysis
}

\author{
Fathalla A. Rihan \\ Department of Mathematical Sciences, College of Science, UAE University, Al Ain, 15551, UAE \\ Email: frihan@uaeu.ac.ae
}

\begin{abstract}
This is a review article to show that delay differential models have a richer mathematical framework (compared with models without memory or after-effects) and a better consistency with biological phenomena such dynamical diseases and cell growth dynamics. The article provides a general computational technique to treat numerically the emerging delay differential models. It introduces the numerical algorithms for parameter estimations, using least squares approach. The article introduces a variational method to evaluate sensitivity of the state variables to small perturbations in the initial conditions and parameters appear in the model. An application to show the consistency of DDE models with cell growth dynamics is also considered.
\end{abstract}

Keywords: DDEs, Epidemiology - SIR, Immunology, Physiology, RK-methods, Time-lags, Parameter estimation, Sensitivity

\section{INTRODUCTION}

A retarded functional differential equation (RFDE) describes a system where the rate of change of state is determined by the present and the past states of the system. If the rate of change of the state depends on its own values as well, the system is called a neutral functional differential equations (NFDEs). When only discrete values of the past have influence on the present rate of change of state, the corresponding mathematical model is either delay differential equation (DDE) or neutral differential equation (NDDE). The theory of RFDEs is of both theoretical and practical interest, as they provide a powerful model of many phenomena in applied sciences such as physics, biology, economics, control theory and so on. They play an important role in explaining many different behaviors. The work reported in [1], [2], [3], [4], [5], [6], [7], [5], [8], [9], [10] indicates the scope for applications of RFDEs in bioscience. The authors remark, therein, how delay differential equations have, prospectively, more interesting dynamics than equations that lack memory effects; in consequence they provide potentially more flexible tools for modelling. However, the numerical treatments of such problems still remains a relatively unexplored area and further research is needed to provide biomodellers with user-friendly adaptive packages.

This paper is organized as follows: Sections $2 \& 3$ introduce the role of delay differential models in dynamic diseases. Section 4 provides most suitable computational techniques for the emerging DDEs. Parameter estimations and sensitivity analysis are given respectively in Sections $5 \& 6$. An applications with cell growth dynamics is given in Section 7, and a conclusion in Section 8.

\section{Delay Models in Dynamic Diseases}

In many applications in the life sciences a delay is introduced when there are some hidden variables and processes, which are not well understood but are known to cause a time-lag [11]. Thus, a delay may in fact represent a reaction chain or a transport process. We shall see later that the mathematical properties of DDEs justify such approximations. A well-known example is Cheyne-Stokes respiration (or periodic breathing), discovered in the 19th century: some people show, under constant conditions, periodic oscillations of breathing frequency [12]. This strange phenomenon is apparently due to a delay caused by cardiac insufficiency in the physiological circuit controlling the carbon dioxide level in the blood. Delays also occur naturally in the chemostat (a laboratory device for controlling the supply of nutrient to a growing population [13]). The use of ODEs to model the chemostat carries the implication that changes occur instantaneously. This is a potential deficiency of the ODE model. There are two sources of delays in the chemostat model: delays due to the possibility that the organizm stores the nutrient (so that the "free" nutrient concentration does not reflect the nutrient available for growth); and delays due to the cell cycle; see [14], [5], [7].

\section{A. Immunology}

The response of an immune system cannot be represented correctly without the hereditary phenomena being taken into account: cell division, differentiation, etc. (the time needed for immune cells to divide, mature, or die). The simple mathematical model of immune response employed by Marchuk [15] describes the interaction of viruses, $V(t)$; antibodies, $F(t)$; plasma cells, $C(t)$; and the relative characteristic of the affected organ, $m(t)$, of a person infected by a viral disease. This model is formulated as a system of four nonlinear DDEs:

$V^{\prime}(t)=\left(p_{1}-p_{2} F(t)\right) V(t)$

$C^{\prime}(t)=\xi(m) p_{3} F(t-\tau) V(t-\tau)-p_{5}\left(C(t)-C^{*}\right)$,

$F^{\prime}(t)=p_{4}(C(t)-F(t))-p_{8} F(t) V(t)$,

$m^{\prime}(t)=p_{6} V(t)-p_{7} m(t)$,

with $t \geq 0$ and $\xi(m)$ is defined by

$$
\xi(m)= \begin{cases}1 & \text { if } m \leq 0.1 \\ (1-m) \frac{10}{9} & \text { if } 0.1 \leq m \leq 1 .\end{cases}
$$

The first equation describes the change in the number of antigen in an organizm (it is a Volterra-Lotka like predatorprey equation). The second equation describes the creation 
of new plasma cells with time-lag due to infection (in the absence of infection, the second term creates an equilibrium at $C(t)=C^{*}$ ). The third equation models the balance of the number of antibody reacting with antigens: the generation of antibodies from plasma cells is described by $p_{4} C(t)$ and their decrease due to aging are described by $\left(-p_{4} F(t)\right)$ and binding with antigens by $\left(-p_{8} F(t) V(t)\right)$. The relative characteristic $m(t)$ of damaging organizm is given by the fourth equation of which the first term expresses the degree of damage to an organ and the second term describes the recuperation due to the recovery activity of the organizm. Finally, the definition of $\xi(m)$ expresses the fact that the creation of plasma cells slows down when the organizm is damaged by the viral infection.

The model (1) has been used to study the relationships between pathogen and the host immune system parameters determining the stability of various steady states. It can also be used to underlying the basic types of infectious disease dynamics: subclinical, acute with recovery, chronic and lethal, or predicting the results of external manipulations with the immune system. In other words, this model allows us, by changing the coefficients $p_{1}, p_{2} \ldots, p_{8}$, to model all sorts of behaviour of stable health, unstable health, acute form of a disease, chronic form etc. (see Marchuk [?]). One of the stationary solutions of (1), that describes the healthy state of an organizm, is

$$
V(t)=0, C(t)=C^{*}, F(t)=F^{*}=C^{*}, \text { and } m(t)=0 .
$$

FIGURES $1 \& 2$ show the solutions of the model (1) (with different parameters) for $\tau=0.5$, with initial values:

$$
V(0)=0.5 \times 10^{-6}, C(0)=1, F(0)=1 \text { and } m(0)=0 ;
$$

and with initial functions:

$$
V(t)=\max \left(0,10^{-6}+t\right), F(t)=1, \quad t \leq 0 .
$$

It may also be noted, from the graphs, that there is either a complete recovery, as in FIGURE 1, or periodic outbreak of the disease, as shown in FIGURE 2 .

Marchuk and his associates [15] developed a hierarchy of immune response models of increasing complexity to account for the various details of defence responses to pathogens. The delays are used in the functional terms describing the proliferation and differentiation of lymphocytes, and represent the time needed for cells to divide, mature (i.e., express certain genes), or to die. Whereas the basic model of an infectious disease has only one time-lag, more sophisticated mathematical models for viral-bacterial infections in lungs, or T-cell division incorporate about ten delays; see [16]. Another example of generic time-lag equations in immunology is provided by Mohler et al. [17] who developed compartmental models for lymphocyte migration. The delays represent the time that cells reside in a particular compartment, or the transit times through compartments, or the duration of inter-compartmental transfer;

It has recently been shown, in a mathematical model for virus dynamics [18], [19], [20], [21], that explicit consideration of the delay between infection of a cell and the production
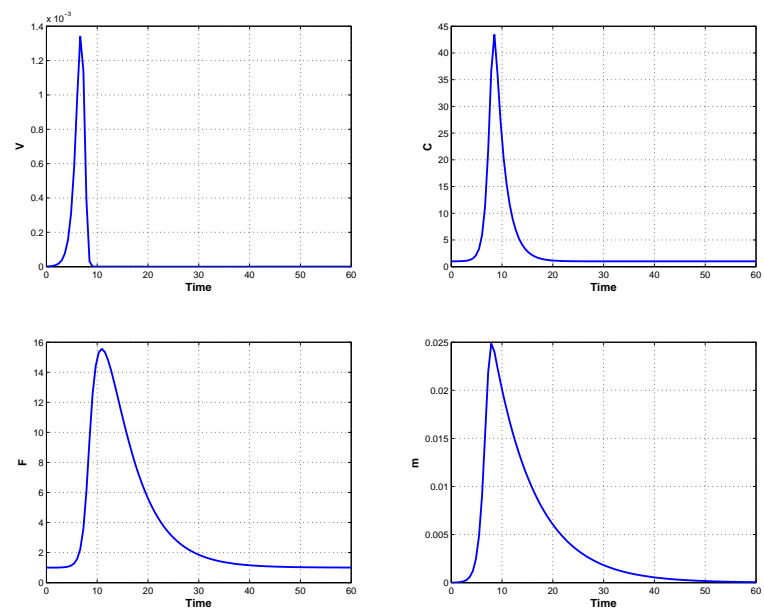

Fig. 1. shows the plot of model (1) for $\tau=0.5$ and $p_{1}=2, p_{2}=0.8$, $p_{3}=10^{4}, p_{4}=0.17, p_{5}=0.5, p_{6}=10, p_{7}=0.12$ and $p_{8}=8$.
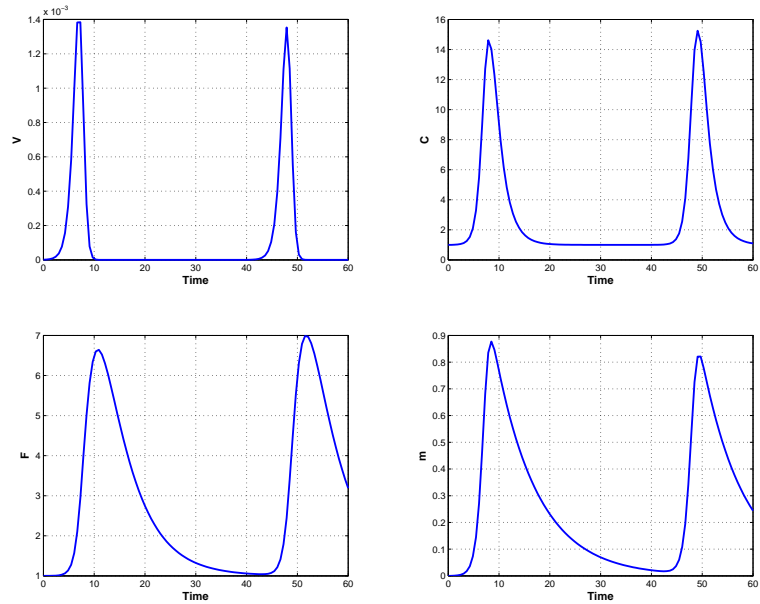

Fig. 2. shows the solution of (1) with the same parameter of 1 except for $p_{6}=300$. The graphs illustrate the periodic outbreak of the disease.

of new viruses is necessary to estimate reliably the turn-over of HIV (Human Immunodeficiency Virus) in infected patients.

The delay differential equations have also long been used in modeling cancer phenomena [22], [23], [24], [25]. In such models the critical time-delay, for which a destabilising Hopf bifurcation of the relevant fixed point occurs, and the conditions on the parameters for such bifurcation are found.

\section{B. Physiology}

The great potential of simple DDEs for capturing complex dynamics observed in physiological systems, was shown in a series of related works by an der Heiden, Mackey et al. [26], [12]. Delay differential equations were used to model unstable patterns of $(i)$ the human respiratory system and regulation of blood concentration of $\mathrm{CO}_{2}$ (periodic breathing and prediction of low- and large-amplitude oscillations), (ii) the production of blood cells (periodic and chaotic regimes), and (iii) hormone regulation in the endocrine system (perioddoubling bifurcations and chaotic solutions); see also [27]. 

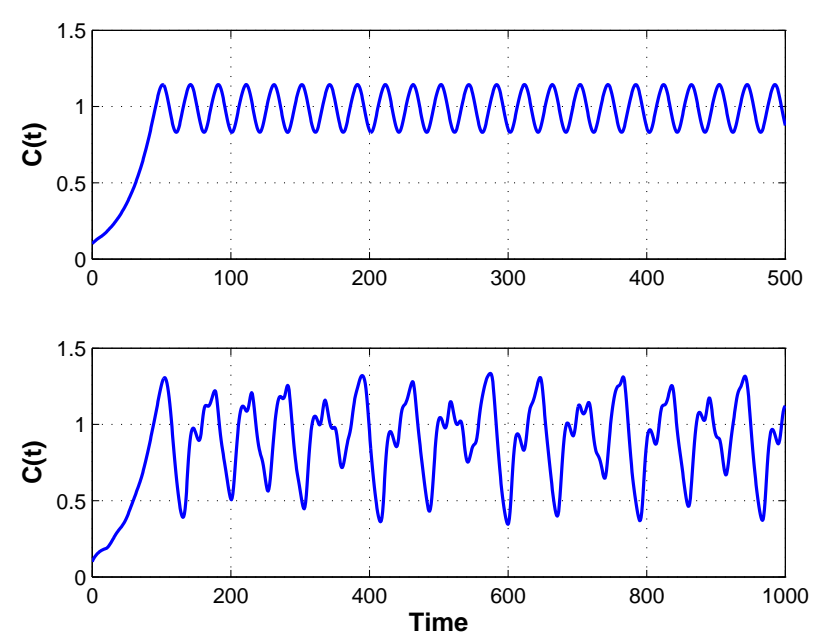

Fig. 3. (top) shows the numerical solution of (2) with parameter values $\alpha=0.1, \gamma=0.1$ days $^{-1}, \lambda=0.2$ days $^{-1}, m=10$ and $\tau=6$ days; (bottom) shows the numerical simulation with the same parameter values as in (a) except an increase in the delay to $\tau=20$ days.

The following model is concerned with the regulation of hematopoiesis, the formation of blood cell elements in the body. For example white and red blood cells, platelets and so on are produced in the bone marrow from where they enter the blood stream. When the level of oxygen in the blood decreases this leads to a release of a substance which in turn cause an increase in the release of the blood elements from the marrow. There is thus a feedback from the blood to the bone marrow.

As an illustrative example, let $c(t)$ be the concentration of cells (the population species) in the circulating blood. We assume that the cells are lost $(=\mathrm{die})$ at a rate proportional to their concentration, that is like $\gamma c(t)$, where the parameter $\gamma$ has dimensions $(\text { day })^{-1}$. After the reduction in cells in the blood stream there is about a 6 day delay before the marrow release further cells to replenish the deficiency (see [12]). We thus assume that the flux $\lambda$ of cells into the blood stream depends on the cell concentration at an earlier time, namely, $c(t-\tau)$, where $\tau$ is the delay. Such assumptions suggest a model equation of the form

$$
\frac{d c(t)}{d t}=\lambda c(t-\tau)-\gamma c(t) .
$$

Glass \& Mackey [28] proposed a possible replacement in the form of the non-linear delay differential equation

$$
\begin{aligned}
\frac{d c(t)}{d t} & =\frac{\lambda a^{m} c(t-\tau)}{a^{m}+c^{m}(t-\tau)}-\gamma c(t), \quad t \geq 0, \\
c(t) & =\alpha, \quad t \leq 0,
\end{aligned}
$$

where $\lambda, a, m, g, \tau$, and $\alpha$ are positive constants. Graphs in FIGURE 3 show the numerical solutions of (2) for two values of the delay time $\tau$.

\section{Epidemiology}

Epidemics have ever been a great concern of human kind, since the impact of infectious diseases on human and animal is enormous, both in terms of suffering and social and economic consequences. This concern is now increased, specially when new swine flu viruses $\mathrm{H} 1 \mathrm{~N} 1^{1}{ }^{1}$ [29] and recently $\mathrm{H} 5 \mathrm{~N} 1$ have sparked a deadly outbreak in some countries and spread into other parts of the world. Mathematical modeling is an essential tool in studying a diverse range of such diseases. The basic elements for the description of infectious diseases have been considered by three epidemiological classes: $S(t)$ that measures the susceptible $e^{2}$ portion of population, $I(t)$ the infected ${ }^{3}$, and $R(t)$ the removed ${ }^{4}$ ones. It was natural to assume that the number of newly infected people per time unit is proportional to the product $S(t) I(t)$. It was also assumed that the number of newly removed persons is proportional to the infected ones, and the total population is a constant $N=S+I+R$ (except death from the disease). KermackMcKendrick [30] thus arrived at the SIR model:

$$
\begin{aligned}
S^{\prime}(t) & =-\beta S(t) I(t), \\
I^{\prime}(t) & =\beta S(t) I(t)-\alpha I(t), \\
R^{\prime}(t) & =\alpha I(t) .
\end{aligned}
$$

Here $\beta$ is the number of contacts between an average infective and the population per unit time (pairwise rate of infection), and $\alpha$ is the fraction of the population which leaves the inflective class (removal rate of infectives). The qualitative analysis is displayed as follows: If $S(0)<\alpha / \beta$, then $I(t)$ is a decreasing function which tends to 0 , and $S(t)$ is also decreasing and tends to a constant level greater than 0 . However, If $S(0)>\alpha / \beta, S(t)$ is also decreasing and tends to a constant level greater than 0 , but $I(t)$ will first increase in a time period $\left(0, T_{0}\right)$, then decrease and tends to 0 after $T_{0}$.

Define a dimensionless quantity $\mathcal{R}_{0}=\beta S(0) / \alpha$, that is a threshold quantity. If we introduce a small number of infectives $I(0)$ into the a susceptible population, then an epidemic will occur if $\mathcal{R}_{0}>1$. As an example, the solution (with all constants equal to one) of (3) (with initial values $S(0)=5, I(0)=0.1, R(0)=0$ ) is plotted in FIGURE 4. We note that an epidemic breaks out, and everybody finally becomes "removed" and nothing further happens.

To prevent an epidemic, we reduce $\mathcal{R}_{0}=\frac{\beta S(0)}{\alpha}$, and maximize the immunization by reducing $I(0)$ and transferring $S(t)$ to $R(t)$ (removed ones). Suppose that $p$ percent of population is successfully immunized, then $S(0)$ is replaced by $(1-p) S(0)$, then $p>1-\frac{\alpha}{\beta S(0)}$. (For practical study to estimate the epidemiological parameters, I refer to [31], [29].)

From the above model, we note that the occurrence of an epidemic depends solely on the number susceptibles, the transmission rate, and recovery rate. In other words, the initial

\footnotetext{
${ }^{1}$ Influenza viruses are defined by two different protein components, known as antigens, on the surface of the virus. They are spike-like features called haemagglutinin $(\mathrm{H})$ and neuraminidase $(\mathrm{N})$ components.

${ }^{2}$ Susceptible: who are not yet infected

${ }^{3}$ Infected: who are infected at time $t$ and are able to spread the disease by contact with susceptible

${ }^{4}$ Removed: who have been infected and then removed from the possibility of being infected again or spreading (Methods of removal: isolation or immunization or recovery or death)
} 


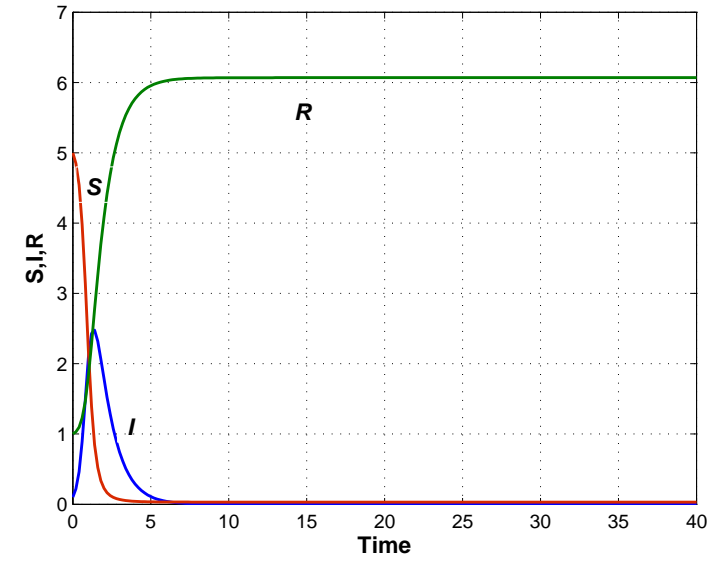

Fig. 4. Solution of SIR model (3) that illustrate the spread of an infection disease in a population.

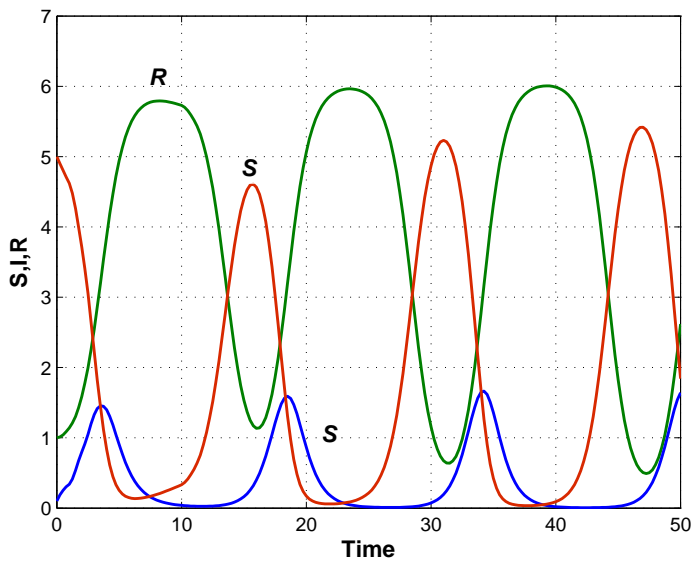

Fig. 5. Solution of model (4) with time delays that displays periodic outbreak of the disease.

number of infectives plays no role in whether or not there is an epidemic. Other considerations, such as vital dynamics (births and deaths), length of immunity, the incubation period of the disease, and disease induced mortality can all have large influences on the course of an outbreak.

1) Development of SIR model (3): The nonautonomous phenomenon occurs mainly due to the seasonal variety, which makes the population behaves periodically [32], [33]. To investigate this kind of phenomenon, in the model, the coefficients should be periodic functions, then the system is called periodic system. Many communicable diseases have this characteristic.

Assume that the immunized people become susceptible again, say after time $\tau_{1}$ (say, $\left.\tau_{1}=10\right)$ (see [34], [35]). If we also introduce an incubation period, $\tau_{2}$, between exposure to infection and becoming infected (say, $\tau_{2}=1$ ), we can arrive at the model

$$
\begin{aligned}
& S^{\prime}(t)=-\beta S(t) I\left(t-\tau_{2}\right)+\gamma I\left(t-\tau_{1}\right), \quad t \geq 0, \\
& I^{\prime}(t)=\beta S(t) I\left(t-\tau_{2}\right)-\alpha I(t), \quad t \geq 0, \\
& R^{\prime}(t)=\alpha I(t)-\gamma I\left(t-\tau_{1}\right), \quad t \geq 0 .
\end{aligned}
$$

The solutions of (4) are shown (with initial functions $[S(t), I(t), R(t)]^{T}=[5,0.1,1]^{T}$ for $\left.t \leq 0\right)$ in FIGURE 5; we note a periodic outbreak of the disease.

2) Development of model (4): If the model allows for a loss of immunity that causes recovered individuals to become susceptible again, we may also consider the more general nonautonomous SIRS epidemic model, with variable periodic coefficients, with distributed delays

$$
\begin{aligned}
S^{\prime}(t)= & \Lambda(t)-\beta(t) S(t) \int_{0}^{\infty} k(\tau) I(t-\tau) d \tau- \\
& m u_{1}(t) S(t)+\xi(t) R(t), \\
I^{\prime}(t)= & \beta(t) S(t) \int_{0}^{\infty} k(\tau) I(t-\tau) d \tau-\left(\mu_{2}(t)+\alpha(t)\right) I(t), \\
R^{\prime}(t)= & \alpha(t) I(t)-\left(\mu_{3}(t)+\xi(t)\right) R(t) .
\end{aligned}
$$

Here $N(t)=S(t)+I(t)+R(t)$ denotes the total number of the population at time $t$. The function $\Lambda(t)$ is the growth rate of the population; function $\beta(t)$ is the daily contact rate, that is the average number of contacts per day; functions $\mu_{1}(t)$, $\mu_{2}(t)$, and $\mu_{3}(t)$ are the instantaneous pro capita mortality rates of susceptible, infective and recovered population, respectively; functions $\alpha(t)$ and $\xi(t)$ are the instantaneous pro capita rates of leaving the infection stage and removed stage, respectively. $k(\tau)$ is the fraction of vector population in which the time taken to become infectious is $\tau$, is assumed to be a nonnegative function on $[0, \infty)$ and satisfies $\int_{0}^{\infty} k(\tau) d \tau=1$ and $\int_{0}^{\infty} \tau k(\tau) d \tau<\infty$.

To analyze the dynamics of the models, numerical methods are necessary, as analytical studies can only provide limited results. We next introduce some reliable computational techniques to solve numerically the emerging delay differential models in biosciences.

\section{General APPROACH FOR SOlving DDEs}

One general approach to the solution of DDEs

$$
\begin{aligned}
y^{\prime}(t) & =f(t, y(t), y(t-\tau)), \quad t \in\left[t_{0}, T\right], \\
y(t) & =\psi(t), \quad t \in\left[-\tau, t_{0}\right] .
\end{aligned}
$$

is based upon the following strategy:

1- Choose a discrete numerical method for solving ODEs;

2- Choose an interpolant $\widehat{y}(t)$ (such as a continuous extension, or a Hermite interpolant) to estimate the numerical solution $\widetilde{y}(t)$ at non-mesh points, then the delayed term $y(t-\tau)$ can be computed at each step;

3- Compute the discontinuity points $\left\{\xi_{k}\right\}$ (one after the other) and, in each interval $\left[\xi_{k-i}, \xi_{k}\right]$, use the chosen numerical ODE method to approximate the solution of the ODE

$$
\begin{aligned}
w^{\prime}(t) & =f(t, w(t), \widehat{y}(t-\tau)), \quad \xi_{k-1} \leq t \leq \xi_{k}, \\
w\left(\xi_{k-1}\right) & =\widehat{y}\left(\xi_{k-1}\right) .
\end{aligned}
$$

The first two steps of this procedure may alternatively be replaced by the direct choice of a continuous numerical method for ODEs (discrete and continuous numerical methods mean the approximation of the solution is on the discrete set of points or in the whole interval of integration, respectively).

As regards stage (3), we may instead seek a method such that the stepsize is determined by the DDE solver (rather than 
the exclusively by the position of the $\left.\left\{\xi_{i}\right\}\right)$. We suppose $\widehat{y}(t)$ has been computed by advancing from the initial point $t_{0}$ : the approximate solution values, $\left\{\widetilde{y}_{n}\right\}$, have been computed on the mesh points, then the solutions at non-mesh points $\widehat{y}(t)$ can be computed for $t_{0} \leq t \leq t_{n}$ using some approximation formula, and for $t<t_{0}$ by evaluation of the initial function. The next step in the numerical solution consists of choosing a stepsize $h_{n}$ such that the solution on the interval $\left(t_{n}, t_{n+1}\right)$ contains no discontinuities, or only "small" discontinuities, in its sufficiently low-order derivatives, and then solving

$$
\begin{aligned}
w^{\prime}(t) & =f(t, w(t), \widehat{y}(t-\tau)), \quad t_{n} \leq t \leq t_{n+1}, \\
w\left(t_{n}\right) & =\widehat{y}\left(t_{n}\right) .
\end{aligned}
$$

In the case of constant lag $\tau$, calculating the numerical solution is straightforward provided that $h_{n} \leq \tau$. For cases where $\tau$ is small relative to the choice of stepsize, or for state-dependent delays in which we find that $\tau\left(t_{*}, \widehat{y}\left(t_{*}\right)\right)<0$ at some points $t_{*}$, it seems there is a need to evaluate $\widehat{y}(t)$ with arguments exceeding the subinterval endpoint $t_{n+1}$ and possibly even exceeding $t_{n+2}$. Given a "discrete" numerical solution, defined on a mesh $\left\{t_{i}\right\}$, there are several methods of extending it to a function of a continuous variable, for example by Lagrange interpolation, or Hermite interpolation or by a continuous extension formula provided by the method itself. The order of accuracy is determined by the error in the interpolation polynomial and the error in the mesh-value $\left\{\widetilde{y}\left(t_{i}\right)\right\}$.

A bifurcation analysis of the system (6) is also used to understand how the solutions and theirs stability change as the parameters in the system vary. In particular, it can be used for the stability, analysis and continuation of equilibria (steadystate solutions), and periodic and quasi-periodic oscillations; See [36], [37].

\section{A. DDE and NDDE solvers and available softwares}

From a modeller's viewpoint, two historical periods in the production of numerical codes for delay equations can be distinguished. During the first period, a number of experimental codes were developed by modellers or numerical analysts. The second period can be characterized by the availability of more sophisticated DDE solvers. The major problems that the designers of such codes try to accommodate are: automatic location or tracking of the discontinuities in the solution or its derivatives, efficient handling of any "stiffness" (if possible), dense output requirements, control strategy for the local and global error underlying the step-size selection, the cost and consistency of interpolation technique for evaluating delayed terms.

The earliest, simple, numerical methods for DDEs (6) utilized the Euler or classical fourth-order $R K$ methods with a constant step-size, supplemented with linear interpolation schemes for the retarded terms. Such adaptations provided minimally effective means for solving models numerically: they had no error control, used fixed step-size, and had problems coping with "stiffness". Numerical analysts are now in a position to cite published algorithms for the numerical solution of DDEs. Several packages and software are available for the numerical integration and/or the study of bifurcations in delay differential equations. Here is a short list for available software:

- Archi (Paul [38]) simulates a large class of functional differential equations.

- DDE23 (Shampine, S. Thompson [39]) simulates retarded differential equations with several fixed discrete delays.

- RADAR5 (Guglielmi, Hairer [40]) simulates stiff problems, including differential-algebraic and neutral delay equations with constant or state-dependent (eventually vanishing) delay.

- DKLAG6 (Thompson [41]) simulates retarded and neutral differential equations with state dependent delays.

- MIDDE (Rihan,et al. [42]) simulates stiff and non-stiff delay differential equations \& Volterra delay integrodifferential equations, using mono-implicit RK methods.

- BIFDD (Hassard [37]) (Fortran 77) normal form analysis of Hopf bifurcations of differential equations with several fixed discrete delays.

- DDE-BIFTOOL (Engelborghs [36]) (MatLab) allows computation and stability analysis of steady state solutions, their fold and Hopf bifurcations and periodic solutions of differential equations with several fixed discrete delays.

For further study of some related issues to the numerical treatments of DDEs, we refer to [7], [4].

\section{Parameter Estimation with DDEs}

Consider even a predictive DDE model of neutral type, parameterized by $\mathbf{p} \in \mathbb{R}^{L}$ which are estimated using a given set of observations,

$$
\begin{aligned}
\mathbf{y}^{\prime}(t) & =\mathbf{f}\left(t, \mathbf{y}(t), \mathbf{y}(t-\tau), \mathbf{y}^{\prime}(t-\tau) ; \mathbf{p}\right), \quad t \in[0, T], \\
\mathbf{y}(t) & =\psi(t, \mathbf{p}), \quad \mathbf{y}^{\prime}(t)=\phi(t, \mathbf{p}), \quad t \in[-\tau, 0] .
\end{aligned}
$$

In (9), the vector function $\mathbf{f}$ is sufficiently smooth with respect to each arguments; $\mathbf{y}(t) \in \mathbb{R}^{M}, \mathbf{y}(t-\tau) \in \mathbb{R}^{M^{\prime}}, \mathbf{p} \in \mathbb{R}^{L}$, and $\tau \in \mathbb{R}^{L^{\prime}}$ is positive constant lag, which may have to be identified as a parameter $\left(L^{\prime} \leq L, M^{\prime} \leq M\right) . \psi(t)$ and $\phi(t)$ are given continuous functions.

Suppose that $N$ observations, $\left\{t_{j} ; Y_{j}^{i}\right\}_{j=1}^{N}$, have been obtained. We are concerned with applying to these data a system of NDDEs (9). The model-fitting problem is then select a value or a set values for $\mathbf{p}$ for which the function $\mathbf{y}(t ; \widehat{\mathbf{p}})$ provides a 'best' fit, at arguments $t=t_{j}$, to the given set $\left\{Y_{j}^{i}\right\}_{j=1}^{N}$ $(1 \leq i \leq M)$. The key part in fitting a model to data is the formulation of the objective function to be optimized that depends on the stochastic features of the errors in the data [43].

There is a variety of methods for regression analysis and interpretation of statistical properties of estimation schemes [44], [45]. The discussion here will be based on the use of weighted least squares (WLS) or a log-least squares (LLS) approach for finding the best-fit parameter values to observed data in the NDDE models. When determining the best fit by 
the WLS process, we suppose that the unknown parameter $\widehat{\mathbf{p}}$ is the value of $\mathbf{p}$ minimizing the weighted objective function:

$$
\Phi_{W}(\mathbf{p})=\sum_{i=1}^{M} \sum_{j=1}^{N}\left[y^{i}\left(t_{j}, \mathbf{p}\right)-Y_{j}^{i}\right]^{2} w_{i j},
$$

where $\mathbf{w}_{j}$ are the weights (possibly related to the accuracy of the data points) $)^{5}$. When $\mathbf{w}_{j}=1$, this is the method of unweighted or ordinary least squares (OLS).

If we adapt the LLS approach, the objective function may take the form

$$
\Phi_{L}(\mathbf{p})=\sum_{i=1}^{M} \sum_{j=1}^{N}\left[\log y^{i}\left(t_{j}, \mathbf{p}\right)-\log Y_{j}^{i}\right]^{2} .
$$

The choice of LLS in model-fitting problem may decrease the exponential nonlinearity of model predictions with respect to p. (It will be assumed that $y^{i}\left(t_{j}, \mathbf{p}\right)>0$.) Another significant feature of the LLS approach is that small relative changes in large data values can be unduly weighted. For comparing between different formulae of objective functions, we refer to Sheiner et al. (1985). (The optimum parameter $\widehat{\mathbf{p}}$ is taken to be the value such that $\Phi(\widehat{\mathbf{p}}) \leq \Phi(\mathbf{p})$, for all physically meaningful values of $\mathbf{p}$ and $\widehat{\mathbf{p}}$.)

When the predictions are governed by models of the form (9), then the least squares (LS) approach (even for models linear in their parameters) usually leads to a nonlinear minimization problem, since the cost function is no longer quadratic. Numerical algorithms for nonlinear LS approach are generally iterative procedures for searching the parameter estimates and require initial starting values. An obvious difficulty is that there is the possibility of the iterative scheme converging to a local minimum, or not converging at all, rather than achieving the desired global minimum. Thus, an appropriate choice of the objective function is a significant factor in determining the ease of solving the parameter estimation problem [46].

Given a set of experimental data, $\left\{Y_{j}\right\}_{j=1}^{N}$, the technique for finding the best-fit parameter values for a given mathematical model and objective function consists of the following steps: (i) Provide an initial guess $\mathbf{p}_{\mathbf{0}}$ for the parameter estimates; (ii) Solve the model equations, using Archi code [38] with the current values of the parameters and calculate the corresponding objective function $\Phi(\mathbf{p})$; (iii) The parameter values are then adjusted (by the minimization routine, for example E04USF ${ }^{6}$ from NAG library; (iv) When no further reduction in the value $\Phi(\mathbf{p})$ is possible, the best fit parameter values have been found; $(v)$ Determine whether the chosen set of parameter values is acceptable (and meaningful) or unacceptable by comparing the objective function value to a given criterion for the objective function or the estimates.

\footnotetext{
${ }^{5}$ The choice of the values $\mathbf{w}_{j}$ is best based on knowledge of the relative precision of the $\mathbf{Y}_{j}$.

${ }^{6} \mathrm{EO}$ UUSF is designed to minimize an arbitrary smooth sum of squares function subject to constraints (which may include simple bounds on the variables, linear constraints and smooth nonlinear constraints) using a sequential quadratic programming (SQP) method.
}

Note that $\Phi(\mathbf{p})$ can have several local minima and that a good code and/or good starting initial parameter values can be of great assistance, both in accelerating the minimization process and finding the global minimum. Local minimum can also be avoided by repeating the iterative scheme for a variety of different initial estimates of parameter vector. We should also draw attention to the fact that, even if the right hand side of (9) and the initial functions are smooth functions, a discontinuity in the first time derivative of the solution appears at time $t_{0}$ and is propagated through the time. The higher derivatives become smoother as time increases. Additional jumps can arise due to discontinuities in the initial functions. These discontinuities propagate into partial derivative of $\Phi(\mathbf{p})$ with respect to $p_{i}$, via solution values $\mathbf{y}(t, \mathbf{p})$. Thus, for correct numerical parameter estimates in DDEs or NDDEs attention should be paid to the position of the jumps and the differentiability of state variable with respect to the time-lag $\tau$.

\section{Sensitivity Analysis}

Of considerable importance in assessing the model (9), is the sensitivity of the model solution $\mathbf{y}(t, \mathbf{p})$ to small variations in the parameter $\mathbf{p}$. For example, if it can be observed that a particular parameter $p_{j}$ has no effect on the solution, it may be possible to eliminate it, at some stage, from the modelling process. We next provide the approach of variational of parameter to evaluate the analysis of sensitivity for DDEs or NDDEs.

\section{A. Variational approach}

The variational approach is to derive, analytically, general sensitivity coefficients for minor changes in the parameters, time delays, and initial data in the model. Use of this approach gives an expression for the sensitivity functions in terms of the solution of an adjoint equation. Variational approach has been used in Rihan (2003) to investigate the qualitative behaviour of the solution of a dynamic system of DDEs due to small variations in the parameters occur in the model. Rihan (2010) extended the approach to include a dynamic system described by a system of NDDEs.

We desire to compute the sensitivity of the state variable $\mathbf{y}(t, \mathbf{p})$ to small variations in the parameters which occur in the NDDE (9). The familiar first-order sensitivity functions for constant parameters $\alpha$, are defined by the partial derivatives $S_{i j}\left(t^{*}\right)=\partial y_{i}\left(t^{*}\right) / \partial \alpha_{j}$, where $\alpha_{j}$ represent the parameters $p_{j}$, the constant lags $\tau$ or the initial values $y_{j}(0)$. Then the total variation in $y_{i}(t)$ due to small variations in the parameters $\alpha_{j}$ is such that

$$
\delta y_{i}(t)=\sum_{j} \frac{\partial y_{i}(t)}{\partial \alpha_{j}} \delta \alpha_{j}+O\left(|\alpha|^{2}\right) .
$$

The functional derivative sensitivity coefficients, however, when the parameters are functions of time such as the initial function, are defined by $\beta_{i j}\left(t, t^{*}\right)=\partial y_{i}\left(t^{*}\right) / \partial \alpha_{j}(t)$ (where 


\begin{tabular}{|l|l|l|l|}
\hline$\rho_{0}$ & $\rho_{1}$ & $\tau$ & $\|$ Error $\|_{2}$ \\
\hline-0.0518 & 0.1054 & 95.33 & 34.41 \\
\hline
\end{tabular}

Parameter estimates for the growth model (17) that best fits data of Figure 6.

$\left.t<t^{*}\right)$. Then the total variation in $y\left(t^{*}\right)$ due to any perturbation in $\alpha(t)$ is denoted by $\delta y\left(t^{*}\right)$, such that:

$$
\delta y_{i}\left(t^{*}\right)=\int_{0}^{t^{*}} \frac{\partial y\left(t^{*}\right)}{\partial \alpha_{j}(t)} \delta \alpha_{j}(t) d t, \quad t<t^{*} .
$$

The functional derivative sensitivity density function $\partial y_{i}\left(t^{*}\right) / \partial \alpha_{j}(t)$ measures the sensitivity of $y_{i}(t)$ at location $t^{*}$ to variation in $\alpha_{j}(t)$ at any location $t<t^{*}$.

For simplicity in equation (9), we write

$$
\begin{aligned}
\mathbf{f}(t) & =\mathbf{f}\left(t, \mathbf{y}(t), \mathbf{y}(t-\tau), \mathbf{y}^{\prime}(t-\tau), \mathbf{p}\right) \\
A^{*}(t) & =\frac{\partial}{\partial \mathbf{y}} \mathbf{f}\left(t, \mathbf{y}(t), \mathbf{y}(t-\tau), \mathbf{y}^{\prime}(t-\tau), \mathbf{p}\right) . \\
B^{*}(t) & \left.=\frac{\partial}{\partial \mathbf{y}_{\tau}} \mathbf{f}\left(t, \mathbf{y}(t), \mathbf{y}(t-\tau), \mathbf{y}^{\prime}(t-\tau), \mathbf{p}\right) .(14), \mathbf{y}^{\prime}(t-\tau), \mathbf{p}\right) . \\
C^{*}(t) & =\frac{\partial}{\partial \mathbf{y}_{\tau}^{\prime}} \mathbf{f}\left(t, \mathbf{y}(t), \mathbf{y}(t-\tau), \mathbf{y}^{\prime}\right. \\
D^{*}(t) & =\frac{\partial}{\partial \mathbf{p}} \mathbf{f}\left(t, \mathbf{y}(t), \mathbf{y}(t-\tau), \mathbf{y}^{\prime}(t-\tau), \mathbf{p}\right) .
\end{aligned}
$$

Theorem 1: If $\mathbf{W}(t)$ is an n-dimensional adjoint function which satisfies the differential equation

$$
\begin{aligned}
\mathbf{W}^{\prime}(t) & =-A^{*}(t)^{T} \mathbf{W}(t)-B^{*}(t)^{T} \mathbf{W}(t+\tau)+ \\
C^{*}(t)^{T} \mathbf{W}^{\prime}(t+\tau), \quad t \leq t^{*}, & \mathbf{W}(t)=\mathbf{W}^{\prime}(t)=0, \quad t>t^{*} ; \\
\mathbf{W}\left(t^{*}\right) & =\left[0, \ldots, 0,1_{i t h}, 0 \ldots, 0\right]^{T}, \mathbf{W}^{\prime}\left(t^{*}\right)=0,
\end{aligned}
$$

then the functional derivative sensitivity functions of NDDEs (9) can be expressed by the formulae

$$
\begin{aligned}
\frac{\partial y_{i}\left(t^{*}\right)}{\partial \mathbf{y}_{0}}=\mathbf{W}(0) & \\
\frac{\partial y_{i}\left(t^{*}\right)}{\partial \mathbf{p}}= & \int_{0}^{t^{*}} \mathbf{W}^{T}(t) D^{*}(t) d t, \quad t \leq t^{*} \\
\frac{\partial y_{i}\left(t^{*}\right)}{\partial \tau}= & -\int_{-\tau}^{t^{*}-\tau} \mathbf{W}^{T}(t+\tau)\left[B^{*}(t+\tau) \mathbf{y}^{\prime}(t)+\right. \\
& \left.C^{*}(t+\tau) \mathbf{y}^{\prime \prime}(t)\right] d t \\
\frac{\partial y_{i}\left(t^{*}\right)}{\partial \psi(t)}= & A^{*}(t+\tau) \mathbf{W}(t+\tau), \quad t \in[-\tau, 0)
\end{aligned}
$$

Proof 1: See Rihan (2010).

\section{ApPlication to CELl GROWTH PROBLEM}

The goal of this section is to apply the above analysis of sections $I V$ and $V$ to fit a time-lag model to the growth of a population of Tetrahymena pyriformis (where the experimental data is given in the Figure 6, and evaluate its sensitivity functions.

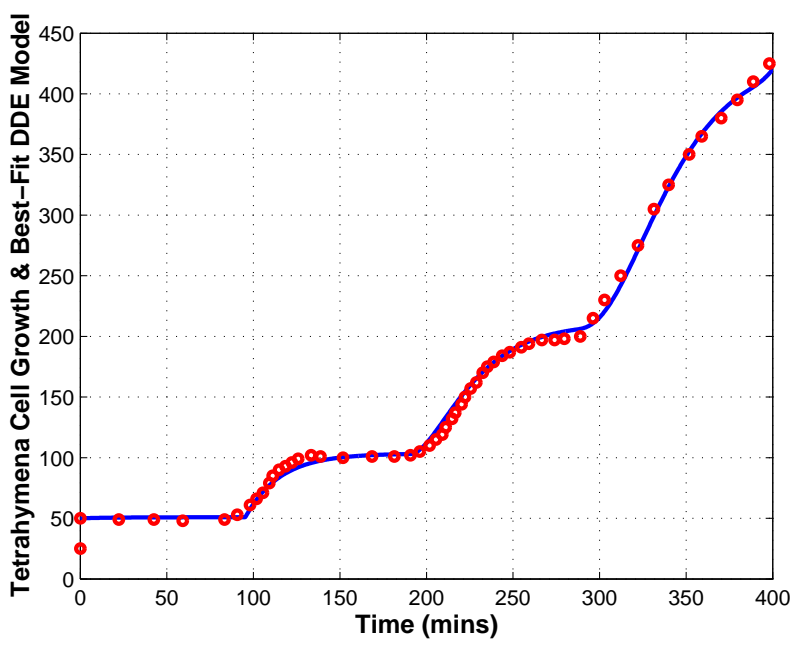

Fig. 6. The circles, $Y_{i}$, represents the data for growth of a population of $Y_{0}=50$ of newborn cells of Tetrahymena pyriformis. This data represents the multiplication of 25 cells in perfect division synchrony at first population doubling. The line, $y(t, p)$, shows the prediction of the perfect model that based on the NDDE (17), with $y(0)=50, y(t)=25$ for $t<0$, and best fit parameters given in Table I. The initially synchronized cell population becomes desynchronized over time.

The cells in the culture of Tetrahymena pyriformis (displayed in Fig. 6) are initially homogeneous and synchronized. This synchronized cell population becomes desynchronized over time. The total observed population as function of time of 50 cells which at time $t=0$ are newborn is shown in Fig. 6. According to the above analysis, we can model this growth by a parameterized linear NDDE

$$
\begin{aligned}
y^{\prime}(t) & =\rho_{0} y(t)+\rho_{1} y(t-\tau)+\rho_{2} y^{\prime}(t-\tau), \quad t \geq 0, \\
y(t) & =\psi(t), y^{\prime}(t)=\psi^{\prime}(t), \quad t \in[-\tau, 0], \quad y(0)=y_{0} .
\end{aligned}
$$

One possible meaning of the parameters of (17) is that $\tau>0$ the average cell-division time; $\rho_{0}<0$ the rate of cell-death in culture; and $\rho_{1}$ the rate of commitment to cell-division process; and $\rho_{2}$ is the gradual dispersal of synchronization of cell-division ( $\rho_{2}=2$ implies pure synchronization). We adopt the Log Least Squares Approach (11) to fit model (17) to the observations given in Figure 6 to estimate the unknown parameters. We consider here a uniform initial function $\psi(t)=25$ for $t \in[-\tau, 0)$, and initial value $y(0)=50$. The graph of Figure 6 displays model prediction for the best fit parameters given in Table I. Prescott (1959) [47] measured the generation times $^{7}$ of a population of Tetrahymena pyriformis cells under uniform conditions. The distribution of generation times in the cell population was displayed for a subpopulation of new born cells at a given time from the synchronized cell population, all of age zero. The mean generation time $\widetilde{\tau}$ was $111 \mathrm{~min}$, which is close to estimated value of the best fit, $\tau=96.33$; see Table I.

${ }^{7}$ Generation time, that varies from cell to cell, is defined as the age at which a cell divides, where age is time measured from birth of a cell. 
We apply the analysis of Section 3 to find analytically the sensitivity functions $\frac{\partial y\left(t^{*}\right)}{\partial \psi(t)} \& \frac{\partial y\left(t^{*}\right)}{\partial \alpha_{i}}\left(t \leq t^{*}\right)$, where $\alpha=$ $\left[\rho_{0}, \rho_{1}, \rho_{2}, y_{0}\right]^{T}$. In (17) $\alpha=\left[\rho_{0}, \rho_{1}, \rho_{2}, y_{0}, \tau\right]^{T}$. The adjoint equation for this case is

$$
\begin{aligned}
W^{\prime}(t) & =-\rho_{0} W(t)-\rho_{1} W(t+\tau)+\rho_{2} W^{\prime}(t+\tau), t \leq t^{*}, \\
W(t) & =0, \quad t>t^{*} ; \quad W\left(t^{*}\right)=1 .
\end{aligned}
$$

The analytical solution of the adjoint Eq (18) is as follows:

(i) $0<t^{*} \leq \tau$

$$
W(t)=e^{-\rho_{0}\left(t-t^{*}\right)}, \quad t \leq t^{*}
$$

(ii) $\tau<t^{*} \leq 2 \tau$

$W(t)=\left\{\begin{array}{l}e^{-\rho_{0}\left(t-t^{*}\right)}-b\left(t-t^{*}+\tau\right) e^{-\rho_{0}\left(t-t^{*}+\tau\right)}, 0<t \leq t^{*} \\ e^{-\rho_{0}\left(t-t^{*}\right)}, t^{*}-\tau<t \leq t^{*}\end{array}\right.$

Here $b=\left(\rho_{1}+\rho_{0} \rho_{2}\right), W(t+\tau)=0$ for $t^{*}-\tau<t \leq t^{*}$ and $W(t+\tau)=e^{-\rho_{0}\left(t-t^{*}+\tau\right)}$ for $0<t \leq t^{*}-\tau$.

The solution of the NDDE (17), with an initial function $\psi(t)=y_{m}\left(\psi^{\prime}(t)=0\right)$, is

$$
y(t)=\left\{\begin{array}{c}
a e^{\rho_{0} t}-y_{m} \xi, 0<t \leq \tau, \\
a e^{\rho_{0} t}-\left[y_{m} \xi-a b(t-\tau)+\right. \\
\left.\quad y_{m} \xi^{2}\right] e^{\rho_{0}(t-\tau)}+y_{m} \xi^{2}, \tau<t \leq 2 \tau,
\end{array}\right.
$$

where $a=\left(y_{0}+y_{m} \xi\right)$, and $\xi=\frac{\rho_{1}}{\rho_{0}}$.

Thus the functional derivative sensitivity density function to the initial function, by using (16d), becomes:

(i) $0<t^{*} \leq \tau$

$\frac{\partial y\left(t^{*}\right)}{\partial \psi(t)}=\rho_{1} W(t+\tau)= \begin{cases}\rho_{1} e^{-\rho_{0}\left(t-t^{*}+\tau\right)}, & -\tau<t \leq t^{*}-\tau \\ 0, & t^{*}-\tau<t \leq 0\end{cases}$

(ii) $\tau<t^{*} \leq 2 \tau$

$\frac{\partial y\left(t^{*}\right)}{\partial \psi(t)}=\left\{\begin{array}{c}\rho_{1} e^{-\rho_{0}\left(t-t^{*}+\tau\right)}- \\ \rho_{1} b\left(t-t^{*}+2 \tau\right) e^{-\rho_{0}\left(t-t^{*}+2 \tau\right)},-\tau<t \leq t^{*}-2 \tau \\ \rho_{1} e^{-\rho_{0}\left(t-t^{*}+\tau\right)}, t^{*}-2 \tau<t \leq 0\end{array}\right.$

While the sensitivity function of $y(t)$ to the initial condition $y(0)$, that given by the formula (16a), is

$\frac{\partial y\left(t^{*}\right)}{\partial y(0)}=W(0)=\left\{\begin{aligned} e^{\rho_{0} t^{*}}, & 0<t^{*} \leq \tau, \\ e^{\rho_{0} t^{*}}+b\left(t^{*}-\tau\right) e^{\rho_{0}\left(t^{*}-\tau\right)}, & \tau<t^{*} \leq 2 \tau .\end{aligned}\right.$

The sensitivity function of $y(t)$ to the constant parameter $\rho_{0}(\equiv$ $\left.\frac{1}{\eta}\right)$, by using (16b), takes the form:

$$
\begin{array}{r}
\frac{\partial y\left(t^{*}\right)}{\partial \rho_{0}}=\int_{0}^{t^{*}} W(t) \frac{\partial F}{\partial \rho_{0}} d t= \\
\begin{cases}\left(a t^{*}-y_{m} \xi \eta\right) e^{\rho_{0} t^{*}}+y_{m} \xi \eta, & 0<t^{*} \leq \tau, \\
\mathbf{I}, & \tau<t^{*} \leq 2 \tau,\end{cases}
\end{array}
$$

where

$$
\begin{aligned}
\mathbf{I}= & \int_{0}^{t^{*}-\tau} W(t) \frac{\partial F}{\partial \rho_{0}} d t+\int_{t^{*}-\tau}^{t^{*}} W(t) \frac{\partial F}{\partial \rho_{0}} d t \\
= & \left(a t^{*}-y_{m} \xi \eta\right) e^{\rho_{0} t^{*}}-2 y_{m} \xi^{2} \eta- \\
& {\left[\left[y_{m} \xi-a b\left(t^{*}-\tau\right)+y_{m} \xi^{2}+a \rho_{2}-b y_{m} \xi \eta\right]\left(t^{*}-\tau\right)\right.} \\
& \left.-y_{m} \xi \eta-2 y_{m} \xi^{2} \eta\right] e^{\rho_{0}\left(t^{*}-\tau\right)}
\end{aligned}
$$

(Similarly, we can deduce $\partial y\left(t^{*}\right) / \partial \rho_{1} \& \partial y\left(t^{*}\right) / \partial \rho_{2}$.) By using $(16 \mathrm{c})$, we obtain the sensitivity of $y(t)$ to small perturbations in the time-lag parameter $\tau$ as:

$$
\frac{\partial y\left(t^{*}\right)}{\partial \tau}=
$$

with $a=\left(y_{0}+y_{m} \xi\right)$ and $b=\left(\rho_{1}+\rho_{0} \rho_{2}\right)$.
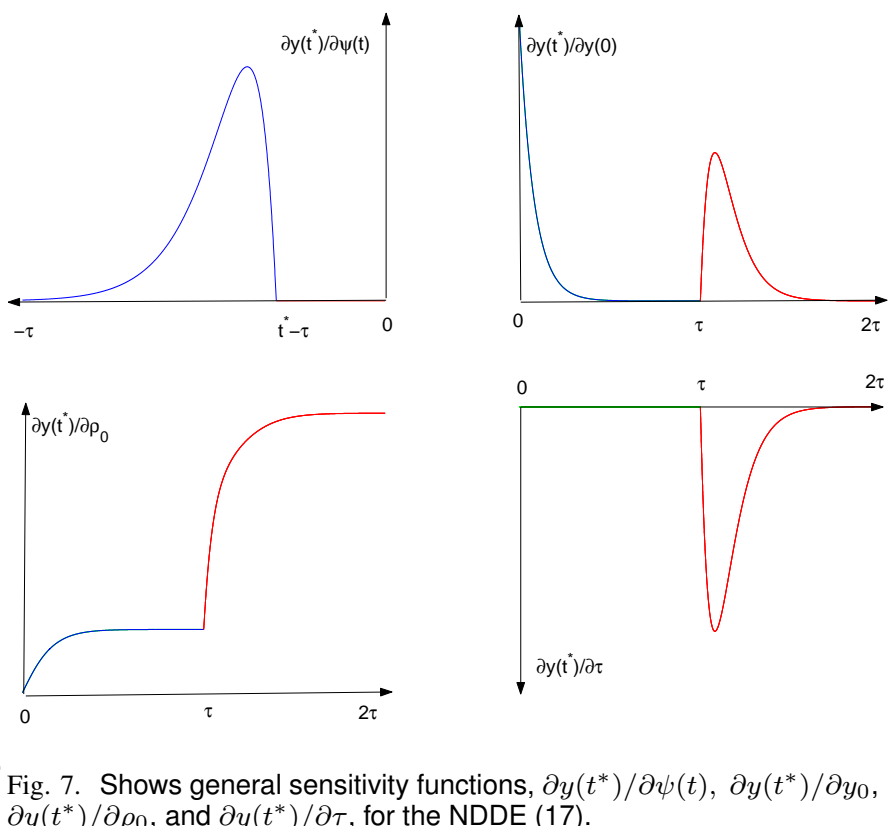

$\partial y\left(t^{*}\right) / \partial \rho_{0}$, and $\partial y\left(t^{*}\right) / \partial \tau$, for the NDDE (17)

We notice from the formula (26) that, as expected, $y(t)$ is sensitive to a change in $\tau$ in the time interval $\tau<t \leq 2 \tau$ and is insensitive to changes in the constant lag $\tau$ in the time interval $[0, \tau]$. The plots (see FIG. 7) have a kink at $t=\tau$ due to the existence of the delay in the system. We may also remark from $\mathrm{Eq}(21)$, that if $y_{0} \neq y_{m}$, then $\partial y\left(t_{i}\right) / \partial \tau$ has a jump at $t_{i}=\tau$. Thereafter attention has to be directed to the objective function when $\tau$ is a parameter to be estimated.

\section{CONCLUDing REMARKS}

Delay differential equations exhibit much more complicated dynamics than ordinary differential equations since a time 
delay could cause a stable equilibrium to become unstable and cause the populations to fluctuate. One requires realistic mathematical models that should be quantitatively and qualitatively consistent with the biological phenomena and experimental data. We have seen that delay models of real-phenomena have more interesting dynamics than equations that lack memoryeffects.

Sensitivity functions clearly demonstrate the measure of the importance of the input parameters. We have remarked how these functions enable one to assess the relevant time intervals for the identification of specific parameters and enhance the understanding of the role played by specific model parameters in describing experimental data.

The literature on this subject is very broad and we cannot quote many interesting papers, as an exhaustive list of references is not possible in this short entry.

\section{ACKNOWLEDGEMENT}

This work is supported by NRF Project (UAE).

\section{REFERENCES}

[1] C. T. H. Baker, G. Bocharov, E. Parmuzin, F. A. Rihan, Some aspects of causal \& neutral equations used in modelling, J. Comput. Appl. Math. 229 (2009) 335-349.

[2] C. T. H. Baker, G. Bocharov, F. A. Rihan, Neutral delay differential equations in the modelling of cell growth, J. Egypt. Math. Soc. 16 (2) (2008) 133-160.

[3] C. T. H. Baker, G. Bocharov, C. A. H. Paul, F. A. Rihan, Models with delay for cell population dynamics: Identification, selection and analysis, Appl. Num. Math. 53 (2005) 107-129.

[4] A. Bellen, M. Zennaro, Numerical Methods for Delay Differential Equations, Oxford University Press, New York, 2003.

[5] G. Bocharov, F. A. Rihan, Numerical modelling in biosciences using delay differential equations, J. Comput. Appl. Math. 125 (2000) 183 199.

[6] F. A. Rihan, Sensitivity analysis of dynamic systems with time lags, J. Comput. Appl. Math. 151 (2003) 445-462.

[7] F. A. Rihan, Numerical Treatment of Delay Dfferential Equation in Bioscience, A PhD thesis submitted to the University of Manchester, 2000.

[8] A. C. Fowler, M. C. Mackey, Relaxation oscillations in a class of delay differential equations, SIAM J. Appl. Math. 63 (2002) 299-323.

[9] N. W. Nelson, A. S. Perelson, Mathematical analysis of delay differential equation models of HIV-1 infection, Math. Biosc. 179 (2002) 73-94.

[10] H. Smith, An Introduction to Delay Differential Equations with Applications to the Life Sciences, Springer: New York Dordrecht Heidelberg London, 2011.

[11] K. Cooke, Z. Grossman, Discrete delays, distributed delays and stability switches, J. Math. Anal. Appl. 86 (1982) 592-624.

[12] M. Mackey, L. Glass, Oscillations and chaos in physiological control systems, Science 197 (1977) 287-289.

[13] H. L. Smith, P. Waltman.

[14] E. Bertta, G. Bischi, F. Solimano, Stability in chemostat equations with delayed nutrient recycling, J. Math. Biol. 28 (1990) 99-111.

[15] G. Marchuk, Mathematical Modelling of Immune Response in Infectious Diseases, Dordrecht: Kluwer Academic Publishers, 1997.

[16] I. A. Sidorov, A. A. Romanyukha, Mathematical modelling of t-cell proliferation, Math. Biosci. 115 (1993) 187-232

[17] Z. H. Faroogi, R. Mohler, Distribution models of recirculating lymphocytes, IEEE. Trans. Biomed. Eng. 36 (1999) 355-362.

[18] K. A. Pawelek, S. Liu, F. Pahlevani, L. Rong, A model of HIV-1 infection with two time delays: mathematical analysis and comparison with patient data, Math. Biosci. 235 (2012) 98109.

[19] R. V. Culshaw, S. Ruan, A delay-differential equation model of HIV infection of CD4 ${ }^{+}$T-cells, Math. Biosci. 165 (2000) 27-39.

[20] K. A. Pawelek, S. Liu, F. Pahlevani, L. Rong, A model of HIV-1 infection with two time delays: mathematical analysis and comparison with patient data, Math. Biosci. 235 (2012) 98-109.
[21] C. Lv, Z. Yuana, Stability analysis of delay differential equation models of HIV-1 therapy for fighting a virus with another virus, J. Math. Anal. Appl. 352 (2009) 672683.

[22] F. A. Rihan, D. H. Abdelrahman, Delay differential model for tumorimmune dynamics with hiv infection of CD4+ T-cells, Int. J. Comput. Math. 90 (3) (2013) 594-614.

[23] M. Bodnar, U. Forys, J. Poleszczuk, Analysis of biochemical reactions models with delays, J. Math. Anal. Appl.

[24] U. Forys, M. Bodnar, J. Poleszczuk, Negativity of delayed induced oscillations in a simple linear dde, Appl. Math. Letters (6) (2011) 982986.

[25] J. Miekisz, J. Poleszczuk, M. Bodnar, U. Forys, Stochastic models of gene expression with delayed degradation, Bull. Math. Biol. 73 (9) (2011) 22312247.

[26] U. an der Heiden, M. Mackey, The dynamics of production and destruction: analytic insight into complex behaviour, J. Math. Biol. 16 (1982) 75-101.

[27] J. J. Batzel, H. Tran, Modelling variation delay in the control system for human respiration: Medical applications, Appl. Math. Copmut. J. 110 (1) (2000) 1-51.

[28] L. Glass, M. Mackey, Pathological conditions resulting from instabilities in physiological control systems, Ann. A.Y. Acad. Sci. 316 (1979) 214 1235.

[29] F. A. Rihan, M.-. N. Anwar, M. Sheek-Hussein, S. Denic, SIR model of swine influenza epidemic in Abu Dhabi: Estimation of Vaccination Requirement, Journal of Public Health Frontier (PHF) 1 (4) (2012) In Press.

[30] W. O. Kermack, A. G. McKendrick, Contributions to the mathematical theory epidemics, Proc. Roy. Soc. A 115 (Part I).

[31] J. Arino, C. S. Bowman, S. M. Moghadas, Antiviral resistance during pandemic influenza: implications for stockpiling and drug use, BMC Infec. Diseases.

[32] T. Zhang, Z.Teng, On a nonautonomous seirs model in epidemiology, Bull. Math. Biol. 69 (2007) 2537-2559.

[33] F. A. Rihan, M. N. Anwar, Qualitative analysis of delayed SIR epidemic model with a saturated incidence rate, Int. J. Diff. Equ. 2012 (2012) 13 pages

[34] C. C. McCluskey, Complete global stability for an SIR epidemic model with delaydistributed or discrete, Nonlinear Anal. Real World Appl. 11 (2010) 55-59.

[35] Y. Takeuchi, W. Ma, E. Beretta, Global asymptotic properties of a delay sir epidemic model with finite incubation times, Nonl. Anal. $42(2000)$ 931-947.

[36] K. Engelborghs, T.Luzyanina, G. Samaey, DDE-BIFTOOL v. 2.00: a Matlab package for bifurcation analysis of delay differential equations.

[37] B. D. Hassard, N. Kazarinoff, Y. Wan, Theory and Applications of Hopf Bifurcation, London Mathematical Society Lecture Note Series 41, Cambridge University Press, Cambridge, 1981.

[38] C. Paul, A user-guide to archi: An explicit runge-kutta code for solving delay and neutral differential equations and parameter estimation problems, MCCM Techical Report 283, University of Manchester (1997).

[39] L. F. Shampine, S. Thompson, Solving ddes in matlab, Appl. Numer. Math. 37 (2001) 441-458.

[40] N. Guglielmi, j. y. v. p. E. Hairer-, title= Implementing Radau II-A methods for stiff delay differential equations.

[41] S. Corwin, D. Sarafyan, S. Thompson, DKLAG6: A code based on continuously embedded sixth-order runge-kutta methods for the solution of state-dependent functional differential equations, Appl. Numer. Math. 24 (1997) 317-330.

[42] F. A. Rihan, E. H. Doha, M. Hassan, N. M. Kamel, Numerical treatments for volterra delay integro-differential equations, Comput. Methods Appl. Math. 9 (3) (2009) 292-308

[43] Y. Bard, Nonlinear parameter estimation, New York, Academic Press, 1974.

[44] H. T. Banks, B. G. Fitzpatrick, Statistical methods for model comparison in parameter estimation problems for distributed systems, J. Math. Biol. 28 (1990) 501-527.

[45] D. Bates, D. Watts, Nonlinear Regression Analysis and its Applications, John Wiley \& Sons, New York, 1988.

[46] D. A. Ratkowsky, Nonlinear Regression Modeling, A Unified Practical Approach, New York: Marcel Dekker, 1983.

[47] D. M. Prescott, Variations in th individial generation times of tetrahymena geleii hs, Exp. Cell Res. 16 (1959) 279-284. 